\section{EMIL ANGEHRN}

\section{Selbstverständigung und Selbsttäuschung}

\section{Zwischen Selbstsein und Selbstverfehlung}

\author{
r. Die Frage der Selbsttäuschung
}

\section{r.I Das Paradox der Selbsttäuschung}

Selbsttäuschung ist ein paradoxer Sachverhalt. Im Prinzip scheint sie unmöglich, und doch stellt sie einen unstrittigen Tatbestand im menschlichen Leben dar. Berühmte literarische Figuren (Alexej Karenin, Homo Faber) verkörpern sie in klassischer Weise. Die Herausforderung für die philosophische Diskussion liegt darin, zu klären, ob und wie wir Selbsttäuschung als einen konsistenten Akt denken können, aber auch, in welcher Weise sie zustande kommt und aus welchen Gründen sie vollzogen wird. Bei keiner dieser Fragen liegt die Antwort auf der Hand. Man kann sogar daran zweifeln, ob es bona fide-Fälle von Selbsttäuschung gibt. Jean-Paul Sartre hat diesen Aspekt betont, indem er das Phänomen der mauvaise foi, das er mit sprechenden Beispielen illustriert, in Termini der Selbstlïge interpretiert und die Auffassung vertritt, dass wir es hier mit einem flüchtigen Phänomen zu tun haben, das gleichwohl eine feste Gestalt annimmt und für viele Menschen einen normalen Teil ihres Lebens darstellt - ein Phänomen, so sein Schluss, das wir weder begreifen noch zurückweisen können.'

Worin aber genau besteht das Problem? Selbsttäuschung, wie sie in Philosophie und Psychologie diskutiert wird, gilt als ein intentionaler Akt. Sich selbst zu täuschen ist nicht dasselbe, wie getäuscht zu werden oder über sich selbst im Irrtum zu sein. Täuschen scheint mit dem Akt des Lügens verwandt, worin der Lügner die Wahrheit, die er anderen verbirgt, kennen muss; selbst im Akt der unbewussten Verstellung, meint Sartre, "muss ich die Wahrheit sehr genau kennen, um sie umso sorgfältiger verbergen zu können «." Wer sich selbst belügt, muss wissen, was er bestreitet oder was er verbirgt. Selbstlüge steh für den eigentümlichen Sachverhalt, dass ich, nach einer Formulierung von Allen W. Wood, "als Opfer der Lüge etwas glauben muss, an das

Jean-Paul Sartre, L'être et le néant, Paris: Gallimard r943, S. 88

2 Jean-Paul Sartre, L'être et le néant, a.a.O., S. 87. ich als Lügner nicht glaube. «" Philosophische Reflexion ist aufgefordert, Selbsttäuschung so zu beschreiben, dass sie die scheinbare Widersprüchlichkeit des Begriffs vermeidet und das Faktum der Selbsttäuschung konsistent, begreifbar - und interessant - werden lässt. ${ }^{4}$

\section{I.2 Die Frage, wie Selbsttäuschung möglich ist}

Die erste Frage, mit der sich die Diskussion befasst, ist zumeist die Frage, wie Selbsttäuschung, trotz ihrer scheinbaren Widersprüchlichkeit, möglich ist. Die naheliegende Antwort besteht darin, unterschiedliche Ebenen oder Dimensionen in der komplexen und undurchschaubaren Verhaltensform der Selbsttäuschung zu unterscheiden. Eine bekannte Strategie ist etwa der psychoanalytische Rekurs auf das Unbewusste, welcher nicht-bewusste Instanzen im Subjekt annimmt, die dem Subjekt bestimmte Erkenntnisse verdecken und falsche Meinungen in ihm erzeugen. So werden der Täuscher und der Getäuschte zu verschiedenen Subjekten. Andere Ansätze ersetzen generell die Idee eines zentralen, einheitlichen Subjekts durch ein Set relativ unabhängiger Subsysteme, so dass die irritierende Figur eines Sich-selbst-Täuschens sich auflöst. Wiederum andere Konzepte bestreiten Sartres Unterstellung, dass der Täuschende wirklich im Bild sein muss über das, was er verbirgt, oder vertreten gar die These, dass der Begriff eines rationalen Handlungssubjekts nicht notwendig verlangt, dass dieses ein reflexives Bewusstsein seiner psychischen oder kognitiven Zustände besitze. ${ }^{5}$ Wir haben ernst zu nehmen, dass Selbsttäuschung durch unbewusste Wünsche und nicht-intendierte Verzerrungen unserer Meinungen bedingt sein kann. Selbstäuschung kann einem wishful thinking entstammen und in einer honest lies resultieren (die sich von der bewussten Lüge unterscheidet und moralisch wie rechtlich anders beurteilt wird als diese). Selbst wenn jemand gegen seine proklamierten Überzeugungen und Präferenzen redet und handelt, agiert

3 Allen W. Wood, "Self-Deception and Bad Faith", in: Brian P. McLaughlin / Amélie Oksenberg Rorty (Hg.), Perspectives on Self-Deception, Berkeley / Los Angeles / London: University of California Press 1988, S. 207-227; hier S. 207.

$4 \mathrm{Vgl}$. Roland Bluhm, Selbsttäuscherische Hoffnung. Eine sprachanalytische Annäherung, Münster: mentis 2012, S. 205-248.

5 Brian P. McLaughlin / Amélie Oksenberg Rorty (Hg.), Perspectives on Self-Deception, a.a.O., S. $2-7$.

6 Vgl. Erik Petry, "Gibt es ein kollektives Gedächtnis? Honest lie und false memory in der Geschichtswissenschaft ", in: Emil Angehrn/Joachim Küchenhoff (Hg.), Das unerledigte Vergangene. Konstellationen der Erinnerung, Weilerswist: Velbrück Wissenschaft 2015 
er nicht notwendig gegen seine wirklichen Überzeugungen und tragenden Haltungen.

1.3 Was in der Selbsttäuschung in Frage steht

Indessen scheinen alle diese Beschreibungen nicht wirklich über das Dilemma der paradoxen Natur der Selbsttäuschung hinauszukommen, nicht wirklich einen rationalen Zugang zu ihr zu eröffnen. Um im Verständnis des Phänomens weiterzukommen, scheint es geboten, die Perspektive zu ändern und die Fragestellung neu zu fassen. Mein Vorschlag ist, sich nicht primär an der Frage, wie Selbsttäuschung möglich ist, zu orientieren, sondern eher daran, wie sie zustande kommt und was ihre Funktion ist. Es ist die Frage nach dem Sinn von Selbsttäuschung, verstanden als Frage, wozu sie gut ist, welches ihr Ort, ihr Stellenwert im menschlichen Leben ist. Offensichtlich spielt Selbsttäuschung eine bedeutende, oftmals zwielichtige, ambivalente Rolle im Leben. In vielen Situationen kann es hilfreich und heilsam sein, sich zu täuschen - über die Welt, über andere, über einen selbst. Menschen können ein Interesse daran haben, falsche Meinungen zu haben, auch wenn sie sich dies nicht zugestehen und möglicherweise sich dessen gar nicht bewusst sind. Illusionen können das Leben erleichtern, sie können von Sorgen und Schuld befreien, Spannungen mindern, unübersichtliche Situationen vereinfachen. Selbsttäuschung ist nicht nur ein Hindernis und ein Irrtum, sie kann ein Mittel sein, das eigene Leben zu ertragen. Sie kann sich als erfolgreiche Strategie der Problemlösung bewähren, auch wenn sie nicht erfolgreiche Strategie der Problemlosung bewähren, auch wen Menschen von erkentüner-)Forderungen entlasten. Sie kann nützlich und pragmatisch sinnvoll, auch human gen entlasten. Sie kann nützlich und pragmatisch sinnvoll, auch human nal, psychologisch dysfunktional und gegebenenfalls moralisch problematisch bleibt.?

Diese Umschreibungen verändern den Blick auf das, was bei der Selbsttäuschung überhaupt in Frage steht. In erster Annäherung schien es so, dass, ähnlich wie bei der Täuschung, falsche Meinungen beziehungsweise die Verhinderung wahrer Erkenntnisse den kritischen Punkt der Selbsttäuschung bilden. Nun zeigt sich, dass dieser Fokus das eigentlich Problematische - dasjenige, was Selbsttäuschung zum theoretischen und praktischen Problem macht - nur unzulänglich erfassen lässt. Natürlich gibt es Selbsttäuschungen dieses Typus. Wir können uns Illusionen darüber hingeben, wie es um unser soziales Ansehen bestellt ist, welches unsere eigenen Fähigkeiten oder auch unsere wahren Gefühle

7 Martin Löw-Beer, Selbsttäuschung, Freiburg/München: Alber 1990, S. 257 ff. sind. Doch scheint im Ganzen des Phänomens wie in relevanten Konstellationen von Selbsttäuschung anderes mit hineinzuspielen, womöglich prioritär zu sein. Selbsttäuschung erschöpft sich nicht in falschen Meinungen über uns selbst, über andere und die Dinge in der Welt. Solche Irrtümer sind im Prinzip durch Hinweis auf relevante Fakten - auf Gegenevidenzen, möglicherweise auch auf Ursachen der Täuschung zu korrigieren. Selbsttäuschung erscheint als ein grundlegenderer Mangel in unserem verstehenden Verhältnis zu uns und zur Welt. Sie ist ein fundamentaleres Verfehlen, das uns selbst, nicht nur unsere kognitiven Zustände betrifft. Näher als der Lüge steht sie dem, was die Existenzphilosophie als Uneigentlichkeit oder existentielle Selbstverfehlung beschreibt. Wenn Selbsttäuschung als Privation gefasst wird, ${ }^{*}$ so ist sie ein Manko nicht nur mit Bezug auf das Erkennen, sondern auch auf das Verstehen, in umfassenderem Sinn mit Bezug auf das Verstehenwollen und das Sich-selbst-Verstehen, letztlich das Selbstsein. Um zu begreifen, inwiefern in solcher Privation ein existentielles Defizit und eine Provokation für die Philosophie liegt, haben wir zuerst zu verdeutlichen, in welchem Sinn das Sein des Menschen mit dem Verstehen und Sichverstehen zusammenhängt.

\section{Selbstsein und Selbstverständigung}

\section{I. Der Mensch als das (sich) verstehende Lebewesen}

Die Frage nach dem Menschen ist für die Philosophie nicht eine Frage neben anderen. Für Kant ist sie die Hauptfrage, auf welche alle Leitfragen der Philosophie - Was kann ich wissen? Was soll ich tun? Was darf ich hoffen? - zurückzuführen sind, so dass man »im Grunde [... alles dieses zur Anthropologie rechnen kann ". Allerdings fällt auf, dass für wichtige Strömungen der modernen Philosophie, so die Hermeneutik und Phänomenologie, die Definition des Menschen nicht einfach der Anthropologie entnommen werden soll. Sie distanzieren sich einerseits von der sogenannten Philosophischen Anthropologie (Scheler, Plessner, Gehlen, Cassirer), die sich auf empirische Forschungen der Human- und Kulturwissenschaften abstützt, andererseits von der metaphysischen Tradition, die von einem vorgegebenen, festen Wesen des Menschen ausgeht. Dagegen begreifen sie den Menschen

8 Kathi Beier, Selbsttäuschung (Grundthemen Philosophie), Berlin / New York: de Gruyter 2010, S. $98 \mathrm{ff}$

9 Immanuel Kant, Logik A 25. 
mit Nietzsche als das "nicht festgestellte Tier $",{ }^{\circ 0}$ das sich seine Bestimmung selbst gibt. Sartre und Heidegger haben diese Sichtweise dahingehend radikalisiert, dass sie die Relation von Essenz und Existenz umkehren" und das "Wassein (essentia) "des Menschen "aus seinem Sein (existentia) " begreifen.' = Sartre begründet diese Umkehrung mit der Freiheit des Menschen, der sich erst setzt und als derjenige entwirft, der er sein wird. Zusätzlich zu diesem Leitmotiv modernen Denkens ist für das phänomenologische Menschenbild ein anderes Motiv zentral.

Der Mensch ist nach Heidegger ein wesentlich verstehendes Wesen, das sich ein bestimmtes Verständnis von sich und der Welt erarbeitet, ein bestimmtes Bild von sich und den Dingen hervorbringt. Sein Verhalten zu sich und zu den Dingen vollzieht sich im Medium dieses Verstehens und ist wesentlich über dieses Verständnis vermittelt. Mensch liches Leben vollzieht sich so, dass der Mensch Selbstbeschreibungen hervorbringt und vermittels ihrer ist, wer er ist. Der Mensch, so die Formel von Charles Taylor, ist das sich selbst interpretierende Tier. ${ }^{13} \mathrm{Da}$ mit ist nicht eine partikulare Eigenschaft, sondern ein Wesensmerkma des Menschen benannt. Nicht ein charakteristisches Merkmal (aufrech ter Gang, Werkzeuggebrauch) oder eine metaphysische Speziesdifferenz (Vernunft, Freiheit) definiert das menschliche Sein, sondern jene spezifische Selbstbeziehung, in welcher der Mensch sich über sich selbs verständigt. Selbstsein vollzieht sich als Hermeneutik des Selbst: ${ }^{4}$ als Bemühen um ein Verständnis seiner selbst in einem vielschichtigen Prozess des Fragens, Erforschens, Entwerfens und Interpretierens.'

Es ist wichtig, den Begriff der Selbstverständigung genau zu fassen. Die kognitive Relation, die hier im Spiel ist, meint mehr als die unmittelbare Selbstpräsenz des Subjekts, die wir als 'Selbstbewusstsein < beschreiben. Sie beinhaltet eine selbsterkenntnis, im Sinne einer inhaltlichen Erkenntnis, die sich auf äußere Eigenschaften, biographische Daten und

ro Friedrich Nietzsche, Jenseits von Gut und Böse, in: Sämtliche Werke. Kritische Studienausgabe in I 5 Bänden, München / Berlin: dtv / de Gruyter I 980 , Bd. 5 , S. $8 \mathrm{I}$.

I I "L'existence précède l'essence «: Jean-Paul Sartre, L'existentialisme est un bumanisme, Paris: Nagel I946, S. 2Iff.

I2 Martin Heidegger, Sein und Zeit, Tübingen: Niemeyer I963, S. 42.

I 3 Charles Taylor, "Self-interpreting animals", in: Philosophical Papers Vol. I, Cambridge: Cambridge University Press 1985, S. 45-76.

14 Der Begriff ist zentral für Paul Ricœur, Soi-même comme un autre, Paris: Seuil 1990.

I5 Vgl. Emil Angehrn, "Selbstsein und Selbstverständigung. Zur Hermeneutik des Selbst «, in: Emil Angehrn / Joachim Küchenhoff (Hg.), Die Vermessung der Seele. Konzepte des Selbst in Philosophie und Psychoanalyse, Weilerswist Velbrück Wissenschaft 2009, S. 163-183. subjektive Haltungen, aber auch auf verdeckte Charakterzüge, geheime Neigungen und verdrängte Wünsche erstrecken kann: auf alles, was die pontane oder methodische Selbsterforschung erkunden kann. Solche Erkenntnis ist keine bloße Feststellung und gesetzmäßige Erklärung. Sie zielt auf ein genuines Verstehen, das die Lebensdaten, Handlungen und Eigenschaften in ihrer Bedeutung erschließen und in ihrem Stellenwert für das, was jemand ist, erfassen will.

Zweierlei spezifiziert dieses Verstehen und Interpretieren seiner selbst. Zum einen die besondere Reflexivität: Es geht um ein Verstehen, das verschieden ist von der objektivierenden Erkenntnis äußerer Sachverhalte, und dies nicht nur insofern, als uns subjektives Erleben in privilegierter Weise aus der Binnenperspektive zugänglich ist. Im Spiel ist die Erste-Person-Perspektive nicht als eine des Feststellens, sondern des Fragens, des Interpretierens und Sichzuschreibens. Sie ist verwandt mit der radikalen Reflexivität ‘, die Charles Taylor dem modernen Selbst zuschreibt und die nach ihm etwa in der antiken Kultur der Selbstsorge noch nicht gegeben ist, deren Praktiken auch aus der Perspektive des Arztes oder Erziehers beschreibbar sind. ${ }^{16}$ Im Gegensatz dazu will der Mensch hier ein Verständnis erlangen, das ihn in seinem Menschsein und in seiner unvertretbaren Einzelheit betrifft - ein Verständnis, das nur er selbst, nicht ein anderer an seiner Stelle suchen, erarbeiten und besitzen kann.

Zum anderen geht es um ein Verstehen, das sowohl ein theoretisches wie ein praktisches Selbstverhältnis meint. Sich in bestimmter Weise zu verstehen, sich als $x$ (als Revolutionär, als gute Mutter, als Fremdling) zu verstehen, kann eine theoretische oder praktische Selbstzuschreibung sein. Mich über mich zu verständigen heißt, über mich Klarheit zu gewinnen in dem, was ich bin und was ich will, woher ich komme und wohin ich gehe, welches meine wahren Bedürfnisse, meine Möglichkeiten und meine Grenzen sind. Selbstverständigung übergreift den Gegensatz von Selbsterkenntnis und Selbstbestimmung, zwischen denen im Leben fließende Übergänge, Verbindungen und Mischungen bestehen. In der konkreten Selbstbeschreibung verschränken sich Formen der Selbstanalyse und der Selbstkritik, der Deutung und des Entwurfs, des Insichgehens und des Ausdrucks. All dies sind Momente einer Reflexion, in der ich mir gegenwärtig, mit mir eins werde - oder mich verfehle.

Darin bestätigt sich, dass das Ziel solcher Selbstverständigung kein rein kognitives ist. Sie strebt nicht allein nach Wissen, sondern nach Sein. Selbstverständigung ist ein Weg der Selbstfindung und der Selbstwerdung. In ihr artikuliert sich ein Seinsstreben, das durch Zielvorstellungen der Ganzheit und der Selbstpräsenz bestimmt ist. In seiner

I6 Charles Taylor, Sources of the Self. The Making of the Modern Identity, Harvard: University Press 1989. 
Selbsterkenntnis und seinem Ausdruck will der Mensch sich finden und mit sich eins werden. Dies schließt keine materiell erschöpfende Vergegenwärtigung ein, und ebenso wenig muss diese ein ganzheitlich-harmonisches Bild des Selbst zeichnen. Nicht die inhaltliche Vollständigkeit ist das Telos, sondern dass das Subjekt in seinem Fragen und Interpretieren zu sich selbst kommt.

\subsection{Grenzen des Verstehens}

Allerdings bietet dieser Weg keine Gewähr, ans Ziel zu gelangen. SelbstAllerdings bietet dieser verständigung kann gelingen oder scheitern. Sie kann ihr zweifaches Ziel der Lücken und Brüchen des Sinns zu tun. Die Hermeneutik des Selbst ist mit dem allgemeinen Problem der Hermeneutik konfrontiert, die immer mit dem Serstehen in Spannung zum Nicht-Verstehen zu tun hat. ${ }^{7}$ In dem Maße, wie das Subjekt sich über sein Wollen und Sein nicht klar werden kann, haftet ihm nicht nur ein kognitives Defizit an. Defizitär bleibt ebenso sein Selbstsein, in welchem es sich nicht als sich selbst entwerfen und als sich selbst finden kann. Die Polarität von Verstehen und Nichtverstehen affiziert das Subjekt in Diem Sem Sichverstehens sind von verschiedener Art. Sie haben ihren Grund teils in der konstitutiven Begrenztheit, teils in kontingenten Behinderungen des Erkennens, teils in Grundproblemen der Existenz.

Sich selbst zu verstehen stößt einerseits auf Grenzen, die in der konstitutiven Schwäche des Erkennens, der Unfähigkeit zur völligen Transparenz und rationalen Durchdringung liegen, aber auch in der strukturellen Unmöglichkeit der restlosen Selbstbeziehung. Es gibt den blinden Fleck der Erkenntnis, der nicht in die Sichtbarkeit einzuholen ist und der a fortiori keine integrale Selbsttransparenz zulässt. Diese strukturelle Grenze der Erkenntnis verhärtet sich durch die Hindernisse, die in der Natur des zu Erkennenden liegen. Die geschichtliche und soziale Bedingtheit, die leibliche und psychische Verfassung verhindern die vollständige Durchsichtigkeit des Selbst für sich selbst. Niemand kann sich in seiner konkreten Existenz voll gegenwärtig werden. Unsere Geschichte, unsere kulturelle Prägung, unsere affektive Stimmung sind uns schichte, unsere kulturelle Pragung, unsere affektive Stimm, wie wir das nur partiell zugänglich. Was wir sind und was wir fühlen, wie wir das geworden sind, was wir sind, ja, was wir selbst meinen und was wir eigentlich wollen, all dies steht uns nicht unverschleiert vor Augen. Nicht

17 Vgl. Emil Angehrn, Sinn und Nicht-Sinn. Das Verstehen des Menschen, Tübingen: Mohr Siebeck zoro. nur alte Texte und ferne Kulturen bilden das Problem der Hermeneutik; wir selbst sind uns fremd und eine Schwelle des Verstehens. Die Psychoanalyse hat dies als die Kränkung formuliert, dass das bewusste Ich nicht Herr im eigenen Hause sei.

Zum anderen kann das Nicht-Verstehen darin gründen, dass sich der Gegenstand selbst dem Verstehen entzieht, ja, geradezu der Verstehbarkeit widerspricht. Irrationale Meinungen können nicht nachvollzogen werden. Unrecht und Gewalt, das Böse und das Leiden gelten seit je als Provokationen für den Anspruch der Vernunft, Wirklichkeit zu begreifen und den Menschen mit der Welt zu versöhnen.

Schließlich radikalisiert sich das Problem des Verstehens, wenn wir die Schwierigkeit nicht nur auf Seiten des zu erkennenden Gegenstandes, sondern auch des verstehenden Subjekts lokalisieren. Der Widerstand gegen die Erkenntnis liegt dann nicht im Dunkel der Sache, sondern im Subjekt selbst. Es geht um eine Selbstverhinderung des Verstehens, für welche das Subjekt verantwortlich ist - sei es aus Trägheit, aus einer inneren Abwehr oder einem selbstverschuldeten Unvermögen. Zuletzt ist es nicht ein Defizit des Verstehenkönnens, sondern des Verstehenwollens, das sich im Extrem in ein Nicht-verstehen-Wollen verkehrt Die Gefährdung der Selbstverständigung liegt dann nicht im Entzug des Selbst für das Verstehen, sondern in der inneren Widersprüchlichkeit des Verstehens selbst. Bedroht ist darin nicht nur das Erkennen, sondern das Sein des Subjekts durch sich selbst. Hinter dem Nicht-verstehen-Wollen öffnet sich der Abgrund des von Kierkegaard beschriebenen Nicht-man-selbst-sein-Wollens. Die negative Zuspitzung der scheiternden Selbstverständigung führt uns zum Problem der Selbsttäuschung zurück.

\section{Selbsttäuschung und Selbstverfehlung}

$$
\text { 3.I. Selbsttäuschung und Willensschwäche }
$$

In alledem erweist sich die Selbsttäuschung als verwandt mit einem anderen Phänomen, das in der Philosophie von alters her diskutiert wird: dem Problem der Willensschwäche. Die Verwandtschaft zeigt sich nicht nur in der paradoxen Struktur eines Sachverhalts, der lebensweltlich vertraut und real verbreitet ist, dessen Möglichkeit aber begrifflich schwer zu fassen ist. Sie zeigt sich im Besonderen auch dann, wenn wir die Selbsttäuschung vor dem Hintergrund der misslingenden Selbstverständigung analysieren und als deren innersten Grund eine Schwäche des Wollens, ja, ein Nicht-Wollen ausmachen. Wir stoßen hier auf einen Aspekt der 
Verbindung zwischen theoretischem und praktischem Selbstverhältnis. Es ist eine Verbindung, die im Positiven wie im Negativen, im gelingenden wie im misslingenden Selbstbezug sichtbar ist und in welcher teils die theoretische, teils die praktische Seite in den Vordergrund rückt. Im vorliegenden Zusammenhang gilt der Akzent der volitiven Seite, dem Wollen als Grund des Selbstseins und des Sichverstehens.

Die Analyse des misslingenden Sichverstehens hat zu einer stufenweisen Vertiefung vom Nicht-Verstehen über das Nicht-verstehen-Wollen zum Sich-nicht-verstehen-Wollen geführt. Sie führt zu einem Nicht-Wollen, welches das Subjekt selbst betrifft, genauer zu einer Widersprüchlichkeit im Wollen des Subjekts, welches gleichzeitig will und nicht will. Zwar kann die Widersprüchlichkeit im Praktischen als weniger problematisch erscheinen denn im Theoretischen, sofern es möglich scheint, entgegengesetzte Strebungen und Willensakte im Subjekt anzunehmen, ${ }^{18}$ nicht aber sich widersprechende Glaubensakte und Behauptungen. Dennoch bildet auch das widersprüchliche Wollen einen existentiellen Irritationspunkt und ein begriffliches Paradoxon. Es geht in der Akrasia nicht um eine Spannung zwischen inhaltlich konkurrierenden Zielen, sondern um eine Gespaltenheit, eine Art performativen Widerspruch in ein und derselben Willensdisposition." Auch in der Selbsttäuschung geht es nicht allein und nicht primär um entgegengesetzte Inhalte - dass wir etwas Bestimmtes meinen oder wollen und uns etwas anderes vormachen -, sondern um eine innere Dissonanz des Wollensakts. Dessen Inkonsistenz verschärft sich im Fall der Selbstverständigung. Dies deshalb, weil das (Nicht-)Wollen hier nicht ein kontingentes Ziel - wie nicht mehr zu rauchen - betrifft, sondern etwas, das dem Menschen unhintergehbar ist. Verstehenwollen und Seinwollen sind Strebensformen, die das Wesen des Menschen ausmachen. Nietzsches Verdacht, dass der Mensch gar nicht an der Wahrheit interessiert sei, oder Heideggers Hinweis, dass der Erkenntnisverzicht der alltäglichen Seinsweise des Menschen entgegenkomme, nennen Gegentendenzen zu einer Grundtendenz, die das Leben als solches charakterisiert und die auf Wissen und Wahrheit gerichtet ist.20

I8 Allen W. Whod ("Self-Deception and Bad Faith", a.a.O., 216) verweist au Zerlinas Arie "Vorrei e non vorrei " in Mozarts Don Giovanni.

59 Eine analoge Figur kennzeichnet Hegels Begriff des Verbrechers, in dessen Tar der Rechtsstatus einer Person negiert und gleichzeitig je schon anerkann wird; entsprechend besteht die Funktion der Strafe (neben der Restitution und Sühne) in der Aufhebung dieser Widersprüchlichkeit.

20 Vgl. Barbara Merker, Selbsttäuschung und Selbsterkenntnis. Zu Heideggers Transformation der Phänomenologie Husserls, Frankfurt am Main: Suhrkamp I 988.
3.2. Existentielle Verfehlung und Selbstentfremdung

Indem im Grunde der Selbsttäuschung eine Verkehrung des Wollens sichtbar wird, erweist sich Selbsttäuschung, jenseits der kognitiven Defizienz, als eine existentielle Schwäche, ein grundlegendes Versagen des Menschen. Es ist eine Verkehrung, in welcher der Mensch mit sich selbst in Zwiespalt gerät, sich selbst fremd wird. Sie nähert sich dem, was die Existenzphilosophie als Uneigentlichkeit thematisiert und was auch andere Theorien als Uneinigkeit des Menschen mit sich, als grundlegende Selbstverfehlung beschreiben. Worin aber besteht diese Verfehlung, was ist ihr Ursprung und was bedeutet sie für die menschliche Existenz?

Nach Heidegger handelt es sich gewissermaßen um den Normalzustand des Menschen, um einen Zustand, in welchem Menschen sich »zunächst und zumeist « befinden. Es ist ein Zustand, in dem sie sowohl ihre praktische Bestimmung eines verantwortlichen Lebens unrealisiert lassen wie sie kognitiv in Täuschungen über sich und die Welt befangen sind Auch wenn Heidegger betont, dass er die Bezeichnung des Alltäglichen oder Uneigentlichen nicht (ab)wertend versteht, ist dessen negativer Charakter, sein Gegensatz zur gesollten, reigentlichen Existenz unübersehbar. Die Frage ist, was dieser Nichtentsprechung, welche in Philosophie und Literatur mannigfach beschrieben wird, zugrunde liegt. Die Grundalternative besteht darin, in ihr entweder ein konstitutives Merkmal oder eine kontingente Beeinträchtigung des menschlichen Seins zu sehen.

Wesentlich zur Existenz gehörig ist sie, sofern sich in ihr die Endlichkeit der conditio humana, die leibliche und psychische, geschichtliche und soziale Bedingtheit des Menschen widerspiegelt. Der Mensch ist nicht reiner Geist, nicht reine Vernunft, nicht reine Erkenntnis. Dass der Mensch unvollkommen und unwahrhaftig existiert, macht seine anthropologische Natur aus. Als nicht-notwendig hingegen erscheint jenes Defizit dort, wo es durch zusätzliche, kontingente Faktoren bedingt ist Ideologiekritik sieht in der Verblendung des Bewusstseins einen Effekt äußerer Herrschaft, die Psychoanalyse untersucht sie als Folge einer innerpsychischen Pathologie. Die Erfahrung realer Negativität, das Erleiden von Krankheit, Gewalt und Unrecht kann das Subjekt der Möglichkeit berauben, mit sich selbst ins Reine zu kommen und die Welt sinnhaft zu verstehen. Geschichts- und kulturphilosophische Theorien situieren die Desintegration des Individuums im Rahmen eines umfassenden - zivilisatorischen, menschheitlichen, seinsgeschichtlichen - Verfalls. Für Heidegger gibt es keine wahre Erkenntnis im Zeitalter der Metaphysik, für Adorno kein richtiges Leben im falschen. Bei alledem aber bleibt die Frage, inwiefern im Subjekt selbst eine Bedingung der Möglichkeit und ein Boden jener Nichtadäquanz und inneren Falschheit auszumachen ist. 
In basaler Weise ist dies dort der Fall, wo die Nichtidentität in die Ontogie des Subjekts eingezeichnet wird. In diesem Sinne vertritt Kathi Beier die These, dass nur eine ontologische, nicht eine epistemologische oder psychologische Explikation das Phänomen der Selbsttäuschung begrifflich "In pointierter Zuspitzung stellt Sartre die onangemessen fassen kann." In pointierter Zuspitzung sellt Sarte die ontologische Fundierung vor Augen. Er geht aus von der Frage, "was der Mensch in seinem Sein sein muss, wenn es ihm möglich sein soll, sich zu verneinen $"{ }^{22}$ und kommt zur Antwort, dass die notwendige Voraussetzung der Unaufrichtigkeit darin besteht, "dass ich ursprünglich gleichzeitig bin und nicht bin, was ich bin « ${ }^{23}$ seine klassische, obstinat wiederholte tig bin und nicht bin, was ich bin "; Grundthese lautet, dass das menschliche Dasem es nicht ist, und nicht ist, was es ist ${ }^{24}$ Man kann sich fragen, wieweit eine es paradoxen Versolche Auskunft das Phan halten wird eine paradoxe Seinsverfassung zugrunde gelegt. Wichtig ist der Charakter der implizierten Negation, die Sartre von Anfang an nicht al bloß konstatierende, sondern als performative Negation auffasst ${ }^{25}$ und die bich in der Unaufrichtigkeit als Negation gegenüber sich selbst, als Flucht vor dem eigenen Sein äußert. Die ontologische Struktur ist Grund dafür vor dem eigenen sein ann, sondern ständig der dass das Bewusstsein nicht nur unaufrichtig sein kann,

Andere Beschreibungen, namentlich diejenige Heideggers, lassen Anderen Gefahr deutlicher hervortreten. Es geht um eine Tendenz, die nicht nur einer ontologischen Schwache, einer Lücke im Sein geschuldet ist, sondern die ethisch besetzt ist und einer Lücke für für das, was er sein will eine Gefahr für den Menschen als solchen, für das, wa er sein soll - und nicht nur für irgendeines seiner Projekte -, bedeutet. Der Mensch besitzt in sich nicht die Stabilität, die ihn te - bedeutet. Der Meidegger durchleuchtet zum Teil einer affirmativen Ordnung mache die ontologisch-ethische Perspektive gewissermaßen auf hren religiösen Hintergrund hin, wenn er stat von einer boß ${ }^{2}$ ) von eine konstitutiven Verfallenstend spricht (auch wenn er betont, dass die Be-

2I Kathi Beier, Selbsttäuschung, a.a.O., S. 5. Der Schlüssel ihrer Explikation Kas Phänomen der Selbsttäuschung logisch, explanatorisch und normativ von einem anderen (dem Begriff der Selbstbestimmung) abhängig ist.

2 Jean-Paul Sartre, L'être et le néant, a.a.O., S. 85

23 Ebd., S. 106.

24 Ebd., S. 97.

25 Ebd., S. 85 .

26 Ebd., S. III.

7 Martin Heidegger, Phänomenologische Interpretationen ausgewählter Abbandlungen des Aristoteles zur Ontologie und Logik, Gesamtausgabe Bd. 62. Frankfurt am Main: Klostermann 2005, S. 356 schreibung nicht religiös verstanden werden müsse).$^{28}$ Die Frage nach dem Grund der Uneigentlichkeit assoziiert sich mit jener nach dem Ursprung des Bösen. An die Stelle der ständigen Gefahr tritt die »ständige Versuchung "zum Verfallen; das Sein des Menschen, so Heidegger, ist wesensmäßig »versucherisch", dem von Kant beschriebenen Hang zum Bösen ausgesetzt. ${ }^{29}$ Auch in der heutigen säkularisierten Welt, meint er, ist das "Tentative « irgendwie da, gehört es untilgbar zum menschlichen Leben..$^{\circ}$ Was die christliche Tradition als Kreislauf von Versuchung, Sündenfall und Erlösung aufspannt, geht in die Tiefenstruktur der Bewegung der Subjektivität ein, in die Polarität von Verfallen und Eigentlichkeit, Verbergung und Wahrheit, Selbstverdeckung und Erschlossenheit. In eigentümlicher Analogie zu der von Sartre zugespitzten Paradoxie wird im Römerbrief die Widersprüchlichkeit des Wollens beschrieben und zugleich ihr Ursprung im Bösen verortet: »Ich aber tue nicht das, was ich will, sondern gerade das, was ich hasse «; "da handle nicht mehr ich, sondern die in mir wohnende Sünde " "3"

Konzeptuell unbefriedigend bleiben solche wie andere dogmatischen Beschreibungen, sofern sie das Negative aus dem Negativen erklären. Die Versuchung weist auf den Teufel als Prinzip des Bösen und Anstifter zum Bösen. Ähnlich verhält es sich, wenn auf die späte Trieblehre Freuds zurückgegriffen und der Todes- und Destruktionstrieb als innerer Kern aufgezeigt wird, der die Selbstverkehrung im wollenden Subjekt bedingt. Es sind Konzepte, welche die explanatorische Lücke füllen sollen, die der Verweis auf die anthropologische Schwäche und Endlichkeit hinterlässt, welche nur die Möglichkeit, nicht die Wirklichkeit des bösen und selbstdestruktiven Verhaltens erklären können. Eine vergleichbare Lücke konstatiert Ricoeur in der Anthropologie der Fehlbarkeit, welche nur die Anlage, nicht den Akt des Bösen begreifen lässt, der theoretisch unableitbar bleibt und nur in einer Reflexion auf die Erzählungen vom Sündenfall und auf die kulturell tradierten Symbole des Bösen zwar nicht in seiner Notwendigkeit begründet, doch in seiner Bedeutung expliziert werden kann 3 .

Eine in gewisser Weise analoge hermeneutische Reflexion lässt sich in Anknüpfung an Kierkegaards und Heideggers Theorie der Angst durchführen. Angst ist darin aufs engste mit dem Problem der Freiheit

28 Martin Heidegger, Phänomenologische Interpretationen zu Aristoteles. Einführung in die phänomenologische Forschung, Gesamtausgabe Bd. 6r, Frankfurt am Main: Klostermann 1985 , S. 154.

29 Martin Heidegger, Sein und Zeit, a.a.O., S. I77.

30 Martin Heidegger, Phänomenologische Interpretationen zu Aristoteles, a.a.O., S. 154 .

31 Röm. 7.15, 7.r.

32 Paul Ricœur, Finitude et culpabilité I: L'homme faillible, II: La symbolique du mal, Paris: Aubier 1960. 
verbunden und zugleich mit dem Ursprung der Verfehlung verknüpft. ${ }^{3}$ Angst entspringt der Konfrontation mit der Unbestimmtheit, mit dem Angst entspringt der Kontrontation mit der. Unbestimmeit, mit dem Nicht-Festgelegtsein der Möglichkeiten und dem Entgleiten der festen Ordnung, und sie ist zuinnerst auf die Haltlosigkeit im Subjekt selbst

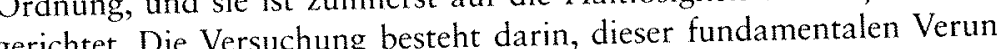
sicherung durch Festlegung zu entkommen. Die Flucht vor der Freiheit ist der Urgrund der Selbstverfehlung, die in die Uneigentlichkeit eingeht. Die ursprüngliche Versuchung zielt darauf, sich in der bestehenden Welt Die ursprüngliche Versuchung zielr darauf, sich in der bestehenden Welt einzurichten, um jener basalen Angst zu entkommen. Das Erfordernis der seigentlichen ' Existenz wäre, die Angst auszuhalten - »in Wahrheit der ${ }^{34}$ - und angesichts von Kontingenz, Unüberzu lernen, Angst zu haben « ${ }^{44}$ - und angesichts von Kontingenz, Unüberne

\section{$3 \cdot 3$ Schluss}

Die Konstellation von Angst und Freiheit ist zurückzubeziehen auf das Problem der Selbsttäuschung. Dass Selbsträuschung vielfach der Angst erwächst, liegt auf der Hand. Selbsttäuschung erscheint phänomenal als eine motivierte, wenn auch nicht intentional bewirkte Verdeckung unangenehmer Sachverhalte. Das Schmerzliche, aber auch das Beschäunangen Anlass sein, die mende, das Bedrohich AnnahAugen vor den Fakten zu verschließen und sich mit falschen Annahmen zu beruhigen. Beruhigung, Entlastung, Erleichterung sind Funktionen der Verdrängung und der Illusionsbildung. Schon die allgemei" ne "Last " des Daseins, das das Dasein seiner Selbsterschlossenheit bewirkt nach Heidegger, dass das Dasein seiner und Schuld im Leben zurechtkommen oder sich in eikretem Leiden und Schuld im Leben zurechtkommen oder sich in einer sinnlosen Welt zurechtfinden muss. Selbsttäuschung erscheint darin als paradigmatischer Fall der Uneigentlichkeit. Sie weigert sich nicht nur, die Herausforderung der Negativität auf sich zu nehmen, sondern leugnet diese und substituiert ihr ein falsches Bild der Welt und des eigenen Selbst. Die Fähigkeit, Brüche und Widersprüche zu ertragen, ist in besonderer Weise gefordert, ja, überfordert, wenn diese nich nur Negativitäten und Inkonsistenzen in der Welt, sondern im eigenen

33 Der Untertitel von Kierkegaards Schrift Der Begriff Angst bezieht diese explizit auf das Problem der Erbsünde.

Wer aber in Wahrheit gelernt hat, Angst zu haben, der wird wie in Tanze

34 "Wer aber in Wahrheit gelernt hat, Angst zu haben, der wird wie in Lehrschreiten, wenn der Endlichkeit Angste auzzuspielen begin Kierkegaard, Der Begriff Angst, Werkausgabe, hg. von Emanuel Hirsch und Hayo Gerdes, DüsBeldorf/Köln: Diederichs I 97 I, Bd. I, S. 348.

seldorf / Köln: Diederichs 197 r, Bd. I, S. 348.
Vgl. Martin Heidegger, Sein und Zeit, a.a.O., S. $134 \mathrm{ff}$.
Leben betreffen. ${ }^{36}$ Selbsttäuschung versucht im Selbst- und Weltverhältnis motivationale und kognitive Kohäsion zu erhalten oder zurückzugewinnen.

Indessen geht es um mehr als um die Bewältigung partikularer Leiden und Widersprüche. Die Tiefenschicht des Phänomens liegt darin, dass Angst nicht irgendwelchen Bedrohungen und Sinnlücken, sondern letztlich dem Abgrund der eigenen Freiheit gilt. Wenn wir die Selbsttäuschung als eine Art Selbstentfremdung betrachten, so liegt die tiefste Entfremdung, die den Menschen bedroht, nicht im Fremdwerden der uns umgebenden Welt und der Trennung von anderen Menschen, sondern im Fremdwerden unserer selbst und zuletzt in der Fremdheit (gegenüber) der eigenen Freiheit. Wir berühren hier einen allgemeinen, anthropologischen Grund der Selbsttäuschung. Im Einzelnen können konkrete Selbsttäuschungen durch ganz verschiede Motive und Lebensumstände bedingt sein, sie können die Folge von Angst oder Scham, von äußerer Not oder seelischem Leiden sein, sie können durch Trägheit, Scheu, Überforderung motiviert sein. Dass sie ihren letzten Grund in einer basaleren Selbstverfehlung haben, zeigt sich nicht zuletzt darin, dass sie im Normalfall nicht durch den Hinweis auf Fakten und Irrtümer korrigiert werden können.

Im Ganzen hat uns der Gedankengang vom Faktum der Selbsttäuschung über das Verstehen und die (misslingende) Selbstverständigung zum Nichtwollen und schließlich zur Angst vor der Freiheit geführt. Von beiden Seiten her ist in diesem Bogen die Verschränkung zwischen der Selbsttäuschung und dem Problem des Selbstseins sichtbar geworden.

Auf der einen Seite ist die Selbsttäuschung von einer kognitiven Selbstintransparenz auf ein tieferliegendes Selbstverhältnis zurückgeführt worden, in einer Nicht-Übereinstimmung und Selbstentfremdung, letztlich einer existentiellen Selbstverfehlung begründet worden. Auf der anderen Seite, gleichsam gegenläufig, hat sich in diesem Verfehlen und Sichfremdwerden das Problem des Verstehens und Sichtäuschens als Horizont und Fokus herausgestellt. Wenn Selbstentfremdung zwar auch in der Entfremdung gegenüber den ursprünglichen Bedürfnissen, gegenüber seinen Pflichten oder der eigenen Natur bestehen kann, so hat sie ihren Kern im Fremdwerden des Sichverstehens, des Willens zur Selbstverständigung. Dieses Sichverstehen liegt jeder anderen Dimension des Sich-zu-sich-Verhaltens, des Sichentwerfens wie des Aneignens seiner Geschichte, seiner Eigenschaften, Gefühle und Strebungen zugrunde. Wenn der Mensch in diesem Willen zur Selbstverständigung bedroht ist, so kommt eine Fremd-

36 Vgl. Daniel Strassberg, "Selbsttäuschungen ", in: Natalie Pieper / Benno Wirz (Hg.), Philosophische Kehrseiten. Eine andere Einleitung in die Pbilosophie, Freiburg / München: Alber 20I4, S. 72-roI. 
heit zum Zuge, die allen anderen Selbstentfremdungen noch vorausliegt. Selbsttäuschung zeigt sich darin nicht nur als ein exemplarischer Fall, sondern als ein innerster Kern existentieller Selbstverfehlung.

\section{MARIA-Sibylla LoTTER}

\section{He that trusteth his own heart is a fool ${ }^{1}$}

Ein ethischer Vergleich antiker und neuzeitlicher Konzepte der Selbstverkennung

\section{Selbsttäuschung: ein epistemisches Phänomen?}

Dass Menschen Dinge, die für sie wichtig sind, nicht immer zur Kenntnis nehmen und aus ihnen die naheliegenden Folgerungen ziehen, ist ein vertrautes Phänomen, das wir als Selbsttäuschung oder auch Selbstbetrug bezeichnen. In der analytischen Philosophie der Gegenwart wird es seit Jahrzehnten debattiert. Man fragt sich, was Selbsttäuschung eigentlich ist, wie sie möglich ist und ob sie überhaupt möglich ist. ${ }^{2}$ Dabei geht man von zwei Varianten der Selbsttäuschung aus: Die einen ${ }^{3}$ verstehen Selbsttäuschung als Weigerung einer Person, sich über wichtige Aspekte ihrer selbst klar zu werden, wobei ihr das, worüber sie sich täuschen möchte, nur in dem Sinne bewusst ist, dass sie sich vor einer näheren Befassung damit fürchtet, weil sie Unangenehmes erfahren könnte. Die anderen begreifen Selbsttäuschung als ein Sich-selbst-Belügen, wobei sich die Person durchaus im Klaren über das ist, worüber sie sich täuschen will; man erklärt sich dies mit einer Art Spaltung der Psyche in zwei Systeme, von denen das eine das andere belügt. ${ }^{4}$

Es ist charakteristisch für diese Diskussionen, dass Selbsttäuschung als ein rein erkenntnistheoretisches Problem behandelt wird: ${ }^{\text {So, als }}$ könne man unabhängig von ethischen und moralischen Gesichtspunk-

I Zitiert nach Joseph Butler, Sermon X "Upon Self-Deceit ", in: Joseph Butler Fifteen Sermons Preached at the Rolls Chapel, London 1969, S. 1.51: And Solomon $[. .$.$] said He that trusteth bis own heart is a fool. This likewise was$ the reason why that precept, Know thyself, was so frequently inculcated by the philosophers of old."

2 So geht etwa Alfred Merle in seinem einflussreichen Buch Self-Deception Unmasked vor, Princeton: Princeton University Press 2001; vgl. S. 3.

3 Vgl. Herbert Fingarette, Self-Deception, London: Routledge 1969; vgl. auch Alfred Mele, Irrationality: An essay on Akrasia, Self-Deception and Self-Control, New York: Oxford University Press r 987

4 Vgl. Donald Davidson, "Täuschung und Teilung «, in: ders., Probleme der Rationalität, Frankfurt: Suhrkamp 2006, S. 332-353.

5 Einen Überblick über neuere Diskussionen bietet der Artikel von Ian Deweese-Boyd, "Self-Deception ", in: Edward N. Zalta (Hg.), The Stanford 


\section{Selbsttäuschung}

Eine Herausforderung für Philosophie und Psychoanalyse

Herausgegeben von

Emil Angehrn und Joachim Küchenhoff 


\section{Inhalt}

Emil Angehrn und Joachim Küchenhoff

Einleitung . . . . . . . . . . . . . . . .

I.

Selbsttäuschung und Selbstverfehlung

Anton Hügli

Selbsttäuschung - und was sie über uns Menschen sagt .....

\section{Emil Angehrn}

Selbstverständigung und Selbsttäuschung.

Zwischen Selbstsein und Selbstverfehlung

Maria-Sibylla Lotter

He that trusteth his own heart is a fool.

Ein ethischer Vergleich antiker und moderner Konzepte

der Selbstverkennung . . . . . . . . . . . . . . . . . . .

II.

Erste Auflage 2017

(1) Velbrück Wissenschaft, Weilerswist 2017 www.velbrueck-wissenschaft.de

Printed in Germany

ISBN 978-3-95832-1. 6-8

Bibliografische Information der Deutschen Nationalbibliothek

Die Deutsche Nationalbibliothek verzeichnet diese Publikation in der

Deutschen Nationalbibliografie; detaillierte bibliografische Daten

sind im Internet über http://dnb.ddb.de abrufbar.

Selbsttäuschung, Subjektkonstitution, Selbstsein

Alice Holzhey-Kunz

Zum Unterschied von snormaler und pathologischer

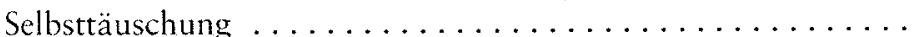

Elfriede Löchel

Lässt sich dem klinischen Konzept des ffalschen Selbst

etwas abgewinnen für eine interdisziplinäre

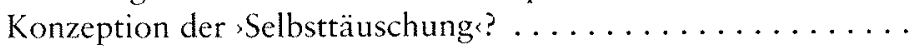

